\title{
Comparison of Two Management Strategies, "Endoscopy First" and "Laparoscopic Cholecystectomy First", for Patients with Gallbladder Stones and Intermediate Risk for Choledocholithiasis: Study Protocol for a Randomized Trial
}

\author{
Ausra Aleknaite ( $\square$ ausra.aleknaite@santa.It) \\ Gintaras Simutis \\ Vilniaus universitetas Medicinos fakultetas \\ Juozas Stanaitis \\ Vilniaus universitetas Medicinos fakultetas \\ Tomas Jucaitis \\ Vilniaus Universitetas \\ Mantas Drungilas \\ Vilniaus Universiteto ligonines Santaros klinikos \\ Jonas Valantinas \\ Vilniaus universitetas Medicinos fakultetas \\ Kestutis Strupas \\ Vilniaus universitetas Medicinos fakultetas
}

Vilnius Unversity, Faculty of Medicine https://orcid.org/0000-0002-2284-3629

\section{Study protocol}

Keywords: choledocholithiasis, endoscopic ultrasound, intraoperative cholangiography, common bile duct stone, endoscopic retrograde cholangiopancreatography, laparoscopic cholecystectomy

Posted Date: May 29th, 2019

DOI: https://doi.org/10.21203/rs.2.9929/v1

License: (a) (i) This work is licensed under a Creative Commons Attribution 4.0 International License. Read Full License 


\section{Abstract}

Background: The optimal approach for patients with gallbladder stones and intermediate risk for choledocholithiasis still remains undetermined. Use of diagnostic endoscopic retrograde cholangiopancreatography should be minimized as it carries considerable risk of post-procedural complications. This study compares two different management strategies: intraoperative cholangiography and endoscopic ultrasound before laparoscopic cholecystectomy for patients with symptomatic cholecystolithiasis and intermediate risk for choledocholithiasis. - Methods: It is a prospective randomized single-centre clinical trial enrolling adult patients undergoing laparoscopic cholecystectomy due to symptomatic gallbladder stones with intermediate risk for choledocholithiasis. The risk for choledocholithiasis is calculated using an original prognostic score - Vilnius University Hospital Index. A total of 106 participants will be included and randomized into two groups. Evaluation of bile ducts using endoscopic ultrasound and endoscopic retrograde cholangiography on demand will be performed before laparoscopic cholecystectomy for one arm ("Endoscopy first"). Intraoperative cholangiography during laparoscopic cholecystectomy and postoperative endoscopic retrograde cholangiopancreatography on demand will be administered in another arm ("Cholecystectomy first"). Postoperative follow-up is 6 months. The primary endpoint is the length of hospital stay. Secondary endpoints will include accuracy of the different management strategies, adverse events of interventions, duct clearance and technical success of interventions (intraoperative cholangiography, endoscopic ultrasound, endoscopic retrograde cholangiography), costs of treatment. • Discussion: This trial is planned determine which strategy is better approach for a patient with intermediate common bile duct stones risk and to define a simple to calculate and safe algorithm on managing choledocholithiasis. • Trial registration: The trial is registered at ClinicalTrials.gov, identification number NCT03658863.

\section{Background}

A gallstone disease can be silent or symptomatic, as well as its most frequent complication choledocholithiasis. About $10 \%$ to $18 \%$ of people undergoing cholecystectomy for gallstones have common bile duct (CBD) stones (1). Untreated choledocholithiasis can lead to acute biliary pancreatitis, acute ascending cholangitis, secondary sclerosing cholangitis, so it is essential to diagnose and treat it on time. Endoscopic retrograde cholangiopancreatography (ERCP) became a prominent diagnostic method for CBD stones since its introduction to clinical practice in 1970s (2). Later on, it was agreed that use of ERCP for diagnostic reasons should be minimized as it carries considerable risk (5 to $10 \%$ ) of postprocedural complications (3). It is noticed that adverse events occur more often to patients with low risk of choledocholithiasis (4). A possibility to avoid using ERCP for diagnostic purposes came with introduction of new diagnostic less invasive procedures, such as magnetic resonance cholangiography (MRCP), endoscopic ultrasound (EUS), intraoperative cholangiography (IOC). Therefore, in the 2000s a discussion about more careful patient selection for ERCP procedure began as it should be left only for those with high probability of demand for therapeutical interventions, i.e. lithectomy, and patients with intermediate risk for choledocholithiasis should undergo additional investigation. 
The most frequently used system to evaluate risk of choledocholithiasis is proposed in 2010 by American Society for Gastrointestinal Endoscopy (ASGE) (5). It already stratifies probability of CBD stones into low, intermediate and high risk categories, and suggests non-invasive investigations for intermediate risk group although its predictive values are not completely satisfying (6-10). These results encourage develop more accurate prognostic systems.

At the Centre of Abdominal Surgery of Vilnius University Hospital Santaros Klinikos an original prognostic index (Vilnius University Hospital index (VUHI)) is used for prediction of choledocholithiasis risk before laparoscopic cholecystectomy (LC). Recently we have evaluated its accuracy and determined new threshold values for low, intermediate and high risk groups (11). The intermediate risk group (risk for choledocholithiasis $25-75 \%$ ) would benefit from additional examination before ERCP. Endoscopic ultrasound (EUS) and intraoperative cholangiography (IOC) are less invasive procedures with high accuracy identifying common bile duct stones. These procedures will be applied for patients with intermediate risk for CBD stones and will help to decide if ERCP is indicated.

We aim to compare LC with IOC to EUS as the first diagnostic biliary intervention in patients with intermediate risk of choledocholithiasis, to evaluate accuracy, technical success and safety of these two management strategies.

The hypothesis to assess is that LC with IOC ("Cholecystectomy first" strategy) will decrease both the length of hospital stay and morbidity by lessening the number of endoscopic investigations (EUS, ERCP) and herewith number of their possible complications, as well as decreasing the complications related to delayed cholecystectomy.

\section{Methods/design}

\section{Design}

This study is a single-centre prospective randomized trial comparing IOC and EUS on finding CBD stones for patients with intermediate risk of choledocholithiasis.

Ethical approval has been obtained from the Vilnius Regional Biomedical Research Ethics Committee, approval protocol number 158200-17-978-473.

\section{Patients}

Flowchart of the patients' enrolment is displayed in Appendix, Figure 1.

\section{Inclusion to the trial}


Eligibility criteria are listed in Appendix, table 1. The trial will enrol 18-80 years old patients with cholecystolithiasis for whom laparoscopic cholecystectomy (LC) is indicated and who has intermediate risk for choledocholithiasis (VUHI 2.6 - 6.9 and one of predictors: dilated common bile duct, elevated total bilirubin or suspected stone in CBD on ultrasound). We will not include pregnant, morbidly obese (body mass index $>40$ ) or severely ill (IV-VI class of American Society of Anesthesiologists physical status classification, contraindications for general anaesthesia or surgery) patients. Also, patients with anastomosis in upper gastrointestinal tract, known or suspected hepatitis of another origin (viral, toxic, etc.), other known cholestatic hepatopancreatobiliary disease will be excluded. We will rule out patients with known complications of gallstone disease, such as biliary pancreatitis, acute cholangitis, and II-III degree acute cholecystitis (as defined in Tokyo guidelines) $(12,13)$.

\section{Elimination from the trial}

Patients will be omitted from the trial if a neoplastic condition will be found in the time of management, if a general status of a participant worsen and he needs urgent interventions not included in the trial protocol, if LC is converted into open cholecystectomy before IOC; if the patient refuses to participate in the trial.

Informed consent will be obtained from all study participants.

\section{Setting}

Participants will be enrolled and the trial will be carried out in Vilnius University Hospital Santaros Klinikos, a tertiary referral centre. The institution has experience in endoscopic ultrasonography, ERCP and intraoperative cholangiography procedures.

\section{Randomization}

Eligible patients who provide informed consent will be assigned to the groups "Endoscopy first" or "Cholecystectomy first" randomly, according to a pre-made sequence. The sequence is generated by a site random.org (Randomness and Integrity Services Ltd). The sequence was created using block randomisation of two elements, A and B (A - "Cholecystectomy first", B - "Endoscopy first") in a ratio 1:1. According to the sequence, sheets with group's name are enclosed to opaque envelopes. Envelopes are numbered and the number of the envelope is patient's number in the trial. When a new participant of the trial is enrolled the topmost envelope is opened by one of investigators and the participant is randomised into the specified group.

\section{Procedure}


The participants of the trial will undergo CBD evaluation depending on the group assignment. For the group "Endoscopy first" EUS is used to evaluate bile ducts. If stones in extrahepatic bile ducts are seen ERCP and lithectomy is performed during the same anaesthesia. LC is performed after endoscopic procedures as soon as possible. In the group "Cholecystectomy first" LC with IOC is performed. If stones are found postoperative ERCP with lithectomy is applied (during cholecystectomy if common bile duct is completely blocked or as soon as possible).

EUS is performed with linear or radial Olympus ultrasound endoscopes. CBD, pancreatic head and adjacent structures are visualised from duodenal bulb and descending duodenum. EUS is considered positive for CBD stone(s) when a constant hyperechogenic lesion with acoustic shadowing is seen in CBD projection.

ERCP procedures are performed by experienced endoscopists (each has more than 5 years of experience in ERCP and more than 500 procedures done). Olympus side-viewing endoscopes TJF-160VR are used. Primary deep selective cannulation of CBD is performed with sphincterotome or cannula and guidewire technique. Diatrizoate (Urografin) and iohexol (Omnipaque) are used as a contrast media. Endoscopic sphincterotomy is performed over a guidewire technique with Olympus pull-type sphincterotome. Papillary balloon dilation using a through-the-scope balloon catheter is applied when stricture is indicated. Stones are removed using a retrieval balloon catheter and/or a Dormia basket. Complete clearance of CBD is documented with a balloon catheter cholangiogram at the end of the procedure. ERCP is considered positive when a filling defect(s) is seen in cholangiogram and/or a stone(s) evacuates from CBD. ERCP is considered unsuccessful when cannulation of bile ducts is technically impossible.

All patients will undergo a standard four-port LC: a $10-\mathrm{mm}$ port at the umbilicus, a $10-\mathrm{mm}$ port at the subxyphoid, a 5-mm port at the bottom of the gallbladder, and a 5-mm port at the right epigastrium. A 30degree laparoscope is used for intra-abdominal visualization. After exposure and identification of the elements of the hepatocystic triangle, a small transverse cut is performed in the cystic duct close to the gallbladder infundibulum with laparoscopic scissors. A 4-French cholangiogram catheter is placed in 5 $\mathrm{mm}$ Cholangiography Fixation Clamp and then inserted into the cystic duct. After verifying the absence of leakage at the catheter insertion site, contrast media (Urografin) diluted in $\mathrm{NaCl} 0.9 \%$ with a $1: 1$ ratio in a $20 \mathrm{ml}$ syringe is injected under fluoroscopic vision (C-arm, Siemens $\mathrm{GmbH}$, Germany). Cholangiograms are assessed by the operating surgeon and radiologist. $\mathrm{IOC}$ is considered positive when there is a filling defect(s) or lack of contrast evacuation to duodenum.

All the procedures of the trial are outlined in Appendix, table 2 (SPIRIT Figure).

\section{Blinding}

As both management strategies - endoscopic evaluation and intraoperative examination - differ in nature and post-procedure effect on a patient, complete blinding of participants is not possible. Before 
enrolment to the trial the participant, treating clinician and investigator will not know to which group participant is assigned.

\section{Follow-up}

The participants are followed while treated inpatient after LC (short term surveillance) and for 6 months after the hospitalisation (long term surveillance). In the short term surveillance period post-procedural adverse events, signs of cholestasis, need for repeated procedures are recorded. In the long term surveillance period participants are encouraged to contact investigators if any symptoms of recurrent cholelithiasis are suspected. 6 months later participants will be contacted via phone or email. Their health status will be evaluated using Medical Outcomes Study Short Form Health Survey (SF-36) (14) with additional questionnaire on possible symptoms of choledocholithiasis (presented in Appendix, Questionnaire 1). If any symptoms of possible gallstone disease are observed the participant is invited for additional investigation (biochemical blood tests, transabdominal ultrasound, MRCP on demand).

\section{Outcomes}

The primary endpoint is the length of hospital stay - duration from enrolment into the trial to discharge, in days.

Secondary endpoints will include:

1) Diagnostic accuracy of the different management strategies (proportion of correctly diagnosed (true positive and true negative) cases in all sample);

2) Technical success of diagnostic and therapeutic biliary procedures (IOC, EUS, ERCP)

a) For IOC: successful cannulation and contrast media injection into CBD;

b) For EUS: successful visualisation of CBD;

c) For ERCP: successful cannulation and contrast media injection into CBD;

d) Successful CBD clearance.

3) Postoperative course and possible complications of treatment:

a) Adverse events of endoscopic interventions and IOC:

i) Bleeding - hematemesis and/or melena or hemoglobin drop >20 g/l;

ii) perforation - evidence of air or luminal contents outside the gastrointestinalf tract; 
iii) post-ERCP pancreatitis - new or worsening abdominal pain persisting for at least $24 \mathrm{~h}$ and requiring analgesics after ERCP in conjunction with an elevation in serum amylase or lipase levels greater than three times the normal upper limit (15), (16);

b) assessment of postoperative course by Clavien - Dindo classification of surgical complications (17).

4) Costs of treatment (charges of diagnostic procedures, invasive procedures, surgery, antibacterial treatment if needed and hospital charges).

\section{Statistical considerations}

Sample size was calculated in reference to collected data on management of choledocholithiasis in the trial centre, Vilnius University Hospital Santaros Klinikos (11). In our previous study mean treatment duration for different management strategy groups (LC-IOC first and ERCP-first) were 5.37 and 7.13 days, with standard deviations 2.5 and 2.8 respectively, these findings were used to calculate requested sample size. a error probability was selected to be 0.05 , power (1- $\beta$ ) 0.095 . Calculated effect size for two independent groups is 0.663 . Required sample size is two groups with 53 participants each.

The endpoints in different management groups will be analysed using chi-square test or T-test for independent means. Two-sided hypotheses are to be checked and a P-value $<0.05$ will be considered statistically significant.

\section{Discussion}

In the era of minimally invasive surgery and personalized medical care the optimal cost-effective strategy for the management of patients with symptomatic gallstones and suspected choledocholithiasis is not categorically defined yet.

The whole approach to patient with gallbladder stones consists of a few steps: evaluation of probability of stones in CBD, visualisation and evacuation of the stones when present and treatment of cholecystolithiasis itself (18). There are a few main clinical dilemmas in the management of choledocholithiasis: which patients should be investigated for CBD stones and what is the optimal way to treat it - single-stage (LC with intraoperative CBD evaluation) or two-stage (preoperative ERCP followed by LC) technique.

First, it is essential to define the criteria for different risk groups. Whilst the majority of recent trials evaluating accuracy of choledocholithiasis prediction refer to ASGE guidelines we performed an analysis of seven different studies on this prognostic system and predictive values of high risk criteria were quite mediocre: general sensitivity was found to be $52.4 \%$, specificity $60.8 \%$, positive predictive value $65.6 \%$, negative predictive value $47.4 \%$, accuracy $55.9 \%$ (11). At the centre of this trial an original prognostic index (VUHI) is used for prediction of choledocholithiasis risk before LC since 1999. It is calculated by formula $\mathrm{VUHI}=\mathrm{A} / 30+0.4 \times \mathrm{B}$, where $\mathrm{A}$ - total bilirubin concentration $(\mathrm{mcmol} / \mathrm{I}), \mathrm{B}-\mathrm{CBD}$ diameter 
measured by ultrasound exam. Results of our previous study showed that VUHI had comparable and, at some parameters, superior performance than the prognostic system of ASGE guidelines (11). The most modest measure was the specificity of VUHI (54\%, whilst sensitivity was $80.5 \%)$. This implied that earlier threshold of the index was kind of weak spot in this evaluation system. Newly generated model for predicted probability of choledocholithiasis sets limits for intermediate risk group, i.e. determines which patients should undergo additional non-interventional investigation. We chose thresholds for intermediate risk group to be at 25 and $75 \%$ of probability for CBD stones considering that upper limit of $50 \%$ as in ASGE guidelines would still leave certain amount of patients who will receive unnecessary ERCPs. Latest EASL (The European Association for the Study of the Liver) guidelines also state that patients with intermediate probability should undergo further evaluation with EUS or MRCP but do not define what this intermediate probability is (19). Meta-analyses showed that these two diagnostic procedures are quite comparable but EUS has better diagnostic accuracy $(20,21)$. Just one trial comparing EUS and IOC was found in PubMed database and showed better predictive values of IOC (22). Considering that this study was performed twenty years ago and imaging technologies have advanced since then it is worth to compare these two methods once again. When comparing IOC with ERCP as a diagnostic procedure, systematic review of ten trials by Gurusamy et al. showed slightly higher sensitivity of IOC with no difference in specificity (23).

The next step is to choose optimal management strategy. In the aforementioned study we assessed effectiveness of different approaches (LC with IOC and ERCP "on demand" versus preoperative ERCP with sphincterotomy and necessary therapeutic interventions followed by LC). Some advantages in both strategies were found: there were less missed stones and false positive cholangiographies in ERCP-first group, meanwhile LC-IOC group had less ERCP related complications and mean length of hospital stay in this group was shorter, in most cases reflecting no need to wait for another procedure (11). Barreras González JE et al. also find these two strategies comparable in efficacy (24). Moreover, meta-analyses of various different trials show that there is no significant difference in the mortality, morbidity, retained stones, and failure rates between the single-stage and two-stage choledocholithiasis management $(1,25)$. The main drawback of preoperative ERCP plus LC strategy against various single-session approaches (intraoperative ERCP, LC with laparoscopic bile duct clearance, open bile duct clearance) is the time: usually there is waiting period between two procedures which prolongs duration of hospital stay and slightly increases the risk to develop recurrent biliary events and cholecystitis $(19,26,27)$. The reduced length of hospital stay was the only significant advantage of intraoperative ERCP found by Cochrane systematic review when comparing single stage and two stages approach in another way - laparoscopic - endoscopic rendezvous versus preoperative endoscopic sphincterotomy (28). A recent meta-analysis by Ricci et al. of four laparoscopic and endoscopic techniques for managing gallstone disease with biliary duct calculi showed that safest and the most successful approach is combined LC with intraoperative ERCP (29). However, one of the biggest limitations to single-session ERCP and LC is difficult coordination of medical personnel, equipment and location of procedure $(30,31)$. Despite these restraints a large survey of general surgeons in USA showed that the majority of respondents preferred ERCP to laparoscopic CBD exploration for the management of choledocholithiasis (32). 
This trial is aimed to answer the question which one of the two strategies - preoperative EUS or LC with $\mathrm{IOC}$ is the optimal solution for intermediate risk of CBD stones. We intend to compare various aspects of two ways of choledocholithiasis management: from accuracy to cost and time efficiency. Thresholds of different risk groups also will be verified prospectively. This trial is planned to define a simple to calculate and safe algorithm on managing choledocholithiasis.

\section{Trial status}

Trial protocol TLA02, version 2.

Trial started on December 15, 2017, anticipated trial completion December 15, 2020.

Trial is registered at ClinicalTrials.gov, identification number NCT03658863.

\section{List Of Abbreviations}

ASGE - American Society for Gastrointestinal Endoscopy

CBD - common bile duct

ERCP - endoscopic retrograde cholangiopancreatography

EUS - endoscopic ultrasound

IOC - intraoperative cholangiography

LC - laparoscopic cholecystectomy

MRCP - magnetic resonance cholangiopancreatography

VUHI - Vilnius University Hospital index

\section{Declarations}

\section{Ethics approval and consent to participate}

Ethical approval has been obtained from the Vilnius Regional Biomedical Research Ethics Committee, approval protocol number 158200-17-978-473.

Informed consent will be obtained from all study participants.

\section{Consent for publication}


Not applicable.

\section{Availability of data and materials}

The datasets generated and/or analysed during the current study are available from the corresponding author on reasonable request.

\section{Competing interests}

The authors declare that they have no competing interests.

\section{Funding}

This trial was conducted with no external funding and is performed on the resources of the hospital as the trial is a part of $\mathrm{PhD}$ research of one of investigators (AA) and the centre of the trial is a university hospital. The approval to carry out the trial on resources of the hospital was obtained and signed as a contract between the hospital and investigators.

\section{Authors' contributions}

GS and AA conceived the project, designed the study, drafted the manuscript, and approved the final submission. GS, AA, JS, TJ and MD arranged inclusion criteria, participates in patients' selection and enrolment. AA performed sample size calculation. KS and JV helped design the study, revised the manuscript, and approved the final submission. All authors read and approved the manuscript.

\section{Acknowledgements}

Not applicable.

\section{References}

1. Dasari B, Tan C, Gurusamy K, Martin D, Kirk G, McKie L, et al. Surgical versus endoscopic treatment of bile duct stones. Cochrane Database Syst Rev [Internet]. 2013 Dec 18 [cited 2017 Jan 10]; Available from: https://www.ncbi.nlm.nih.gov/pubmed/24338858

2. Costi R. Diagnosis and management of choledocholithiasis in the golden age of imaging, endoscopy and laparoscopy. World J Gastroenterol. 2014;20(37):13382. 
3. Freeman ML. Complications of Endoscopic Retrograde Cholangiopancreatography. Gastrointest Endosc Clin N Am. 2012 Jul;22(3):567-86.

4. Williams EJ, Green J, Beckingham I, Parks R, Martin D, Lombard M. Guidelines on the management of common bile duct stones (CBDS). Gut. 2008 Feb 27;57(7):1004-21.

5. Maple JT, Ben-Menachem T, Anderson MA, Appalaneni V, Banerjee S, Cash BD, et al. The role of endoscopy in the evaluation of suspected choledocholithiasis. Gastrointest Endosc. 2010 Jan;71(1):1-9.

6. Adams MA, Hosmer AE, Wamsteker EJ, Anderson MA, Elta GH, Kubiliun NM, et al. Predicting the likelihood of a persistent bile duct stone in patients with suspected choledocholithiasis: Accuracy of existing guidelines and the impact of laboratory trends. Gastrointest Endosc. 2015 Jul;82(1):88-93.

7. Prachayakul V, Aswakul P, Bhunthumkomol P, Deesomsak M. Diagnostic yield of endoscopic ultrasonography in patients with intermediate or high likelihood of choledocholithiasis: a retrospective study from one university-based endoscopy center. BMC Gastroenterol [Internet]. 2014 Dec [cited 2017 Feb 22];14(1). Available from: http://bmcgastroenterol.biomedcentral.com/articles/10.1186/1471-230X$14-165$

8. Suarez AL, LaBarre NT, Cotton PB, Payne KM, Coté GA, Elmunzer BJ. An assessment of existing risk stratification guidelines for the evaluation of patients with suspected choledocholithiasis. Surg Endosc. 2016 Feb 19;30(10):4613-8.

9. Sethi S, Wang F, Korson AS, Krishnan S, Berzin TM, Chuttani R, et al. Prospective assessment of consensus criteria for evaluation of patients with suspected choledocholithiasis. Dig Endosc. $2015 \mathrm{Jul}$ 27;28(1):75-82.

10. Rubin MIN, Thosani NC, Tanikella R, Wolf DS, Fallon MB, Lukens FJ. Endoscopic retrograde cholangiopancreatography for suspected choledocholithiasis: Testing the current guidelines. Dig Liver Dis. 2013 Sep;45(9):744-9.

11. Aleknaite A, Simutis G, Stanaitis J, Valantinas J, Strupas K. Risk assessment of choledocholithiasis prior to laparoscopic cholecystectomy and its management options. United Eur Gastroenterol J. 2018 Apr;6(3):428-38.

12. Kiriyama S, Takada T, Strasberg SM, Solomkin JS, Mayumi T, Pitt HA, et al. TG13 guidelines for diagnosis and severity grading of acute cholangitis (with videos). J Hepato-Biliary-Pancreat Sci. 2013 Jan;20(1):24-34.

13. Yokoe M, Takada T, Strasberg SM, Solomkin JS, Mayumi T, Gomi H, et al. TG13 diagnostic criteria and severity grading of acute cholecystitis (with videos). J Hepato-Biliary-Pancreat Sci. 2013 Jan;20(1):3546. 
14. Brazier JE, Harper R, Jones NM, O'Cathain A, Thomas KJ, Usherwood T, et al. Validating the SF-36 health survey questionnaire: new outcome measure for primary care. BMJ. 1992 Jul 18;305(6846):1604.

15. Cotton PB, Lehman G, Vennes J, Geenen JE, Russell RC, Meyers WC, et al. Endoscopic sphincterotomy complications and their management: an attempt at consensus. Gastrointest Endosc. 1991 Jun;37(3):383-93.

16. Cotton PB, Eisen GM, Aabakken L, Baron TH, Hutter MM, Jacobson BC, et al. A lexicon for endoscopic adverse events: report of an ASGE workshop. Gastrointest Endosc. 2010 Mar;71(3):446-54.

17. Dindo D, Demartines N, Clavien P-A. Classification of surgical complications: a new proposal with evaluation in a cohort of 6336 patients and results of a survey. Ann Surg. 2004 Aug;240(2):205-13.

18. Almadi MA, Barkun JS, Barkun AN. Management of suspected stones in the common bile duct. Can Med Assoc J. 2012 May 15;184(8):884-92.

19. EASL Clinical Practice Guidelines on the prevention, diagnosis and treatment of gallstones. J Hepatol. 2016 Jul;65(1):146-81.

20. Meeralam Y, Al-Shammari K, Yaghoobi M. Diagnostic accuracy of EUS compared with MRCP in detecting choledocholithiasis: a meta-analysis of diagnostic test accuracy in head-to-head studies. Gastrointest Endosc. 2017 Dec;86(6):986-93.

21. Giljaca V, Gurusamy K, Takwoingi Y, Higgie D, Poropat G, Štimac D, et al. Endoscopic ultrasound versus magnetic resonance cholangiopancreatography for common bile duct stones. Cochrane Database Syst Rev [Internet]. 2015 Feb 27 [cited 2017 Jan 10]; Available from:

https://www.ncbi.nlm.nih.gov/pubmed/25719224

22. Montariol T, Msika S, Charlier A, Rey C, Bataille N, Hay JM, et al. Diagnosis of asymptomatic common bile duct stones: preoperative endoscopic ultrasonography versus intraoperative cholangiography--a multicenter, prospective controlled study. French Associations for Surgical Research. Surgery. 1998 Jul;124(1):6-13.

23. Gurusamy KS, Giljaca V, Takwoingi Y, Higgie D, Poropat G, Štimac D, et al. Endoscopic retrograde cholangiopancreatography versus intraoperative cholangiography for diagnosis of common bile duct stones. Cochrane Hepato-Biliary Group, editor. Cochrane Database Syst Rev [Internet]. 2015 Feb 26 [cited 2018 Nov 26]; Available from: http://doi.wiley.com/10.1002/14651858.CD010339.pub2

24. Barreras González JE, Torres Peña R, Ruiz Torres J, Martínez Alfonso MÁ, Brizuela Quintanilla R, Morera Pérez M. Endoscopic versus laparoscopic treatment for choledocholithiasis: a prospective randomized controlled trial. Endosc Int Open. 2016 Nov;4(11):E1188-93. 
25. Lu J. Two-stage vs single-stage management for concomitant gallstones and common bile duct stones. World J Gastroenterol. 2012;18(24):3156.

26. Kageoka M, Watanabe F, Maruyama Y, Nagata $K$, Ohata A, Noda Y, et al. LONG-TERM PROGNOSIS OF PATIENTS AFTER ENDOSCOPIC SPHINCTEROTOMY FOR CHOLEDOCHOLITHIASIS. Dig Endosc. 2009 Jul;21(3):170-5.

27. Byrne MF, McLoughlin MT, Mitchell RM, Gerke H, Pappas TN, Branch MS, et al. The fate of patients who undergo "preoperative" ERCP to clear known or suspected bile duct stones. Surg Endosc. 2009 Jan;23(1):74-9.

28. Vettoretto N, Arezzo A, Famiglietti F, Cirocchi R, Moja L, Morino M. Laparoscopic-endoscopic rendezvous versus preoperative endoscopic sphincterotomy in people undergoing laparoscopic cholecystectomy for stones in the gallbladder and bile duct. Cochrane Hepato-Biliary Group, editor. Cochrane Database Syst Rev [Internet]. 2018 Apr 11 [cited 2018 Nov 30]; Available from: http://doi.wiley.com/10.1002/14651858.CD010507.pub2

29. Ricci C, Pagano N, Taffurelli G, Pacilio CA, Migliori M, Bazzoli F, et al. Comparison of Efficacy and Safety of 4 Combinations of Laparoscopic and Intraoperative Techniques for Management of Gallstone Disease With Biliary Duct Calculi: A Systematic Review and Network Meta-analysis. JAMA Surg. 2018 Jul 18;153(7):e181167.

30. EIGeidie AA. Single-session minimally invasive management of common bile duct stones. World J Gastroenterol. 2014;20(41):15144.

31. Mallick R, Rank K, Ronstrom C, Amateau SK, Arain M, Attam R, et al. Single-session laparoscopic cholecystectomy and ERCP: a valid option for the management of choledocholithiasis. Gastrointest Endosc. 2016 Oct;84(4):639-45.

32. Baucom RB, Feurer ID, Shelton JS, Kummerow K, Holzman MD, Poulose BK. Surgeons, ERCP, and laparoscopic common bile duct exploration: do we need a standard approach for common bile duct stones? Surg Endosc. 2016 Feb;30(2):414-23.

33. Banks PA, Bollen TL, Dervenis C, Gooszen HG, Johnson CD, Sarr MG, et al. Classification of acute pancreatitis-2012: revision of the Atlanta classification and definitions by international consensus. Gut. 2013 Jan;62(1):102-11.

\section{Appendix}

Table 1. Eligibility criteria 
Inclusion

Criteria

Age 18-80 years

Symptomatic cholecystolithiasis

Intermediate risk for choledocholithiasis (VUHI 2,6 - 6,9 and one of predictors: dilated common bile duct $>6 \mathrm{~mm}$, elevated total bilirubin $>21 \mathrm{mcmol} / \mathrm{l}$ or suspected stone in CBD on ultrasound).

\section{Exclusion}

Criteria

acute cholangitis, as defined in Tokyo guidelines 2013 (12);

moderately severe or severe biliary pancreatitis as defined in revised Atlanta classification (33)

acute cholecystitis, degree II-III by Tokyo guidelines 2013 (13);

anastomosis in upper gastrointestinal tract;

known cholestatic hepatopancreatobiliary disease;

known or suspected hepatitis (viral, toxic, etc.);

contraindications for general anaesthesia or surgery;

IV-VI class of ASA physical status classification;

morbid obesity (body mass index $>40$ );

pregnancy;

patient's refusal to participate in the study.

Table 2. Enrolment, interventions and surveillance procedures 
Study

period

Enrolment Allocation Post-

allocation

$\begin{array}{lll}\text { Management } & \begin{array}{l}\text { Short term } \\ \text { surveillance }\end{array} & \begin{array}{l}\text { Long term } \\ \text { surveillance }\end{array} \\ \text { (inpatient) } & \text { (6 months) }\end{array}$

\section{Recruitment:}

Eligibility screen $\quad \mathrm{x}$

Informed consent $\quad \mathrm{x}$

Randomisation $\quad \mathrm{x}$

Management

strategy:

"Endoscopy first" x

"Cholecystectomy X

first"

Assessments:

Bilirubin $\quad \mathrm{x} \quad$ on demand on demand

concentration

Ultrasound $\quad \mathrm{x} \quad$ on demand on demand

$\begin{array}{lll}\text { Surgical records } & \mathrm{x} & \mathrm{x} \\ \begin{array}{l}\text { Postoperative } \\ \text { records }\end{array} & \mathrm{x} & \mathrm{x}\end{array}$

Interview with

patient

$x$

Questionnaire 1. Symptoms of possible choledocholithiasis

You had a gallbladder removal operation performed in Santaros Klinikos 6 months ago. You have consented to participate in the clinical study on risk of bile duct stones. Please answer to following questions on your health status after the operation.

Have you had any pain in the upper right part of your abdomen or in other areas? If yes, please specify.

Have you become jaundiced (have you noticed that your skin or eyes turned yellow)?

Have you had fever without any clear reason (e.g., common cold)? 
Have you had doctor appointment for any of those reasons?

If yes, what investigations were performed and what diagnosis was stated?

If you had any of those symptoms, would you agree to arrive at Santaros Klinikos for additional investigation - abdominal ultrasound and blood tests?

\section{Figures}




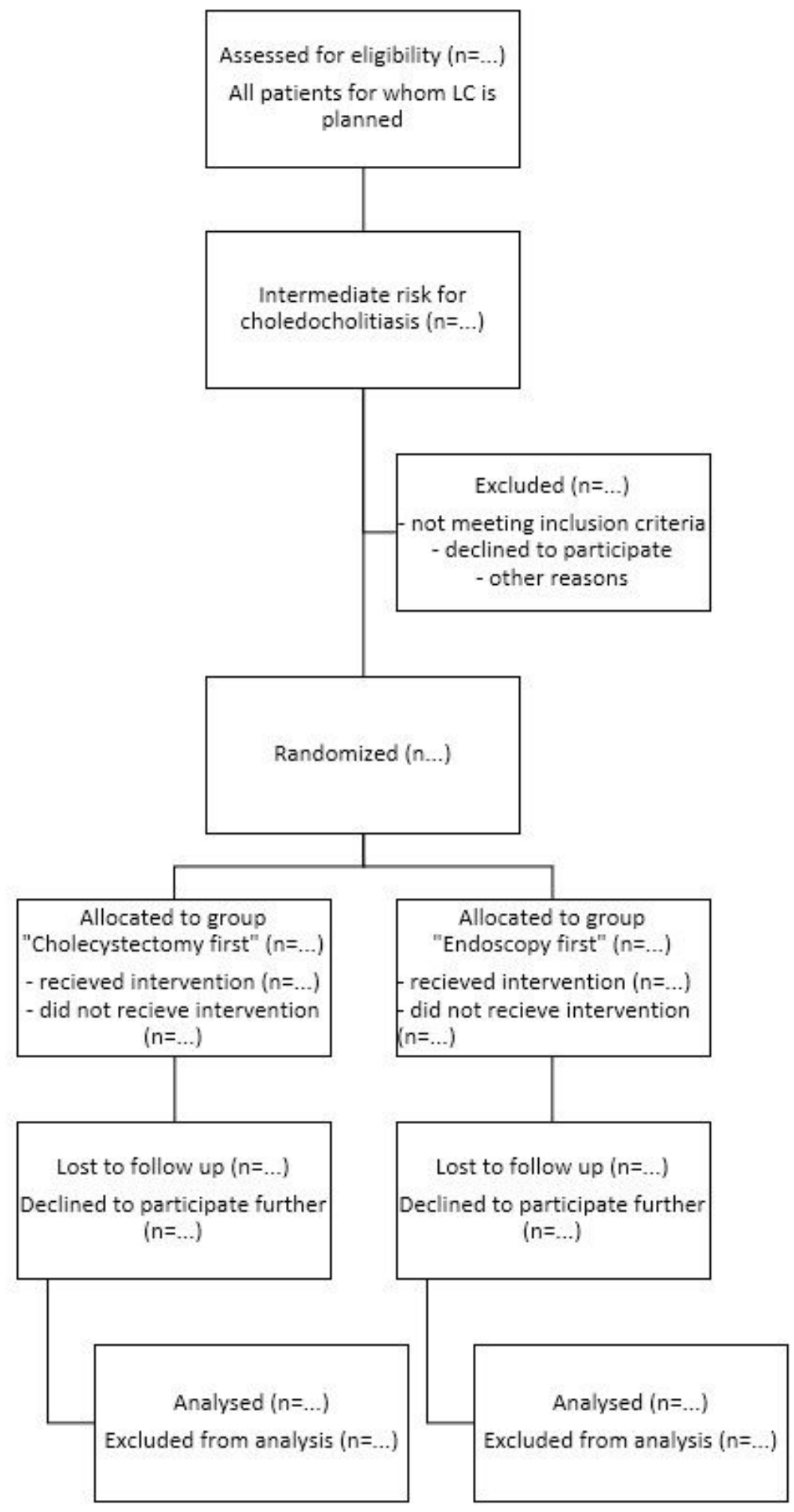

\section{Figure 1}

Flowchart of the study.

\section{Supplementary Files}

This is a list of supplementary files associated with this preprint. Click to download. 
- supplement1.doc

Page 18/18 